\section{REVISITING POLYPHARMACY IN THE HOSPICE IN-PATIENT SETTING}

Shahlaa Walsh, Emily Sills. Woking and Sam Beare Hospices

10.1136/bmjspcare-2019-ASP.147

Background Polypharmacy continues to be a concern in the palliative care population. Potentially Inappropriate Medications (PIMs), with no short-term benefit, contribute to pill burden, poor concordance and adverse drug events (ADEs). An initial study, carried out in two hospice in-patient units found that $40 \%$ of patients were discharged on 10 or more medications and $83 \%$ were prescribed at least one PIM. A deprescribing tool was implemented as part of a polypharmacy quality improvement project, based on Lindsey et al's guideline for deprescribing unnecessary medications in the palliative population.

Aim To identify whether the introduction of a deprescribing tool, to aid doctors in discussions about rationalising PIMs, reduces the number of unnecessary medications and total number of medications on discharge.

Method 63 inpatient notes (21 discharges, 42 deaths) were audited using the original audit proforma. For discharges; the number of medications pre and post admission were recorded. Additionally, the number of PIMs prescribed and the number of medications rationalised on admission were recorded.

Standards remained the same as the original study: $80 \%$ of medications with limited benefit to be stopped and $80 \%$ of patients to be discharged on 7 medications or less.

Results $71 \%$ of all patients were on at least one PIM preadmission compared to $83 \%$ previously (pre-tool). $43 \%$ of patients were discharged on 7 or less medications, an increase from $38 \%$ in the initial audit. There was a 50\% reduction in the total number of PIMs prescribed from admission to discharge. $65 \%$ patients had at least one drug rationalised on admission. Only $66 \%$ of patients went home on at least 1 PIM compared to nearly $80 \%$ in the initial audit.

Conclusion The deprescribing tool is beneficial in helping prescribers to recognise polypharmacy and consider rationalising medications, specifically PIMs, in hospice inpatients.

\section{5 'BETTER THE LETTER' - IMPROVING HOSPITAL DISCHARGE COMMUNICATION FOR PALLIATIVE CARE PATIENTS: A SERVICE EVALUATION}

Sophie Hancock, Maria Debattista, Valerie O'Donnell. Lancashire Teaching Hospitals NHS Foundation Trust

\subsection{6/bmjspcare-2019-ASP.148}

Background Communication between hospital and primary care at discharge is vital to ensure patients feel supported and prevent crisis readmissions. The hospital palliative care team (PCT) have developed comprehensive training for all doctors and produced 'better the letter'guidance to improve discharge letter content. The most recent innovation is the inclusion of a specific palliative care section on discharge letters with key information for GPs around advance care planning.

Methods A 'snapshot'service evaluation was conducted from October to November 2018. Discharge letters were examined for patients discharged from hospital who were on the hospital PCT caseload. Discharge letters were examined for presence of a completed palliative care section and relevant GP actions. Electronic records were reviewed for suitable patients for the presence and content of an EPaCCS (electronic palliative care coordination system) record, created by the GP within two weeks of discharge.

Results 166 discharged patients were identified and 151 of these patients had discharge letters available for evaluation and were included in the study. $74 \%$ of included patients had an active palliative care section on their discharge letter. EPaCCS could not be assessed for 51 of the 151 patients due to patient death following discharge. $60 \%$ of patients with an active palliative care section on the discharge letter had an active EPaCCS record versus $38 \%$ of patients without an active palliative care section.

Conclusions There is some evidence that the presence of a specific palliative care section on discharge letters improves the creation of EPaCCS records in the community, which may improve the coordination of care for these patients. However, there was a proportion of missing data due to death soon after discharge. Further work is needed to improve communication of palliative care information on hospital discharge, and evaluation of the impact this has on patient care.

\section{USING PALLIATIVE PERFORMANCE SCALE (PPS) AND PHASE OF ILLNESS TOOL (POI) TO FACILITATE IMPROVEMENT IN COMMUNICATION OF PALLIATIVE CARE NEEDS ACROSS HEALTH AND SOCIAL CARE}

Claire Howie, Marianne MacLeod, Monica Keenan, Kim Steel. NHS Fife

\subsection{6/bmjspcare-2019-ASP.149}

Background In 2018, the NHS Fife Specialist Palliative Care team were awarded funding to spread the learning form the Macmillan funded TCAT 'Best Supportive Care in Lung Cancer' project. Communication barriers and imprecise language existed across our health care environments was one of the factors that had been identified within the Lung Cancer Best Supportive Care project, that needed improvement and this second phase of the project gave us this opportunity.

Methods

- Process map the 'hand-offs'in communication between professionals that occur from the bedside assessment in hospital to having a professional assessing again in normal place of residence;

- Teaching and feedback at all points identified in the processmap;

- Quantitative assessment of how many patients discharged by palliative care occupational therapy team were re-admitted within 14 days and how many died in normal place of residence;

- Qualitative interviews with professionals involved at the discrete points of the process map to describe the changes made to practice.

Results

- There was a change in response from the discharge assessment team;

- More patients were discharged home and died in their normal place of residences;

- There was an increase in the number of discharges home of patients known to the Specialist Palliative Care Occupational Team; 